\title{
Guest Editorial: Nanophotonics in Japan
}

\author{
Motoichi Ohtsu \\ University of Tokyo, Department of Electronics Engineering, Tokyo 113-8656, Japan \\ ohtsu@ee.t.u-tokyo.ac.jp
}

Makoto Naruse

National Institute of Information and Communications Technology, New Generation Network

Research Center, Photonic Network Group, 4-2-1 Nukui-kita, Koganei, Tokyo 184-8795,

Japan

naruse@nict.go.jp

and

University of Tokyo, School of Engineering, Department of Electronics Engineering,

Building 9, 2-11-16 Yayoi, Bunkyo-ku, Tokyo 113-8656, Japan

naruse@ee.t.u-tokyo.ac.jp

Nanophotonics is a novel technology that utilizes the optical near-field, which is the electromagnetic field that mediates the interaction between nanometric particles located in close proximity to each other. Novel devices, fabrication techniques, systems, and sensing have been developed in this regard. By utilizing the intrinsic nature of the optical near-field energy transfer and subsequent dissipation, some of these developments have realized novel functions and phenomena that were impossible using propagating light. They constitute examples of "qualitative innovation" in optical technology. Research and development in nanophotonics originated in Japan, where it has been active for the last two decades, and several national projects have been organized.

This special section entitled "Nanophotonics in Japan" was organized to introduce recent activities in this field. It consists of a review paper and original, contributed papers. As some of the contributed papers are still being reviewed at the time of this writing, they will be published in the special section after this process has been completed. 